University of Nebraska - Lincoln

DigitalCommons@University of Nebraska - Lincoln

May 1992

\title{
MULTIPLET FINE STRUCTURE OF THE Gd AND Tb 5P LEVELS
}

Dongqi Li

Syracuse University

Peter A. Dowben

University of Nebraska-Lincoln, pdowben@unl.edu

M. Onellion

University of Wisconsin, Madison

Follow this and additional works at: https://digitalcommons.unl.edu/physicsdowben

Part of the Physics Commons

Li, Dongqi; Dowben, Peter A.; and Onellion, M., "MULTIPLET FINE STRUCTURE OF THE Gd AND Tb 5P LEVELS" (1992). Peter Dowben Publications. 155.

https://digitalcommons.unl.edu/physicsdowben/155

This Article is brought to you for free and open access by the Research Papers in Physics and Astronomy at DigitalCommons@University of Nebraska - Lincoln. It has been accepted for inclusion in Peter Dowben Publications by an authorized administrator of DigitalCommons@University of Nebraska - Lincoln. 


\title{
MULTIPLET FINE STRUCTURE OF THE Gd AND Tb 5P LEVELS
}

\author{
Dongqi $\mathrm{Li}$ and P.A.Dowben \\ Department of Physics, Syracuse University, Syracuse, NY 13244-1130 \\ M.Onellion \\ Department of Physics, University of Wisconsin, Madison, WI 53706
}

\begin{abstract}
Fine structure is observed in the photoemission of Gd and Tb $5 p$ levels as a result of multiplet splitting. These splittings are the consequence of different $\mathrm{J}$ final states that occur as a result of interactions with partly filled $4 \mathrm{f}$ and/or $5 \mathrm{~d}$ levels.
\end{abstract}

\section{INTRODUCTION}

Photoemission from shallow core levels has been employed to probe magnetic ordering(1-7]. Recently we have investigated the rare earth $5 p$ levels in different photoemission geometries to study rare earth thin film magnetism[3,4]. A model for the photoemission shallow core level fine structure is important to the understanding of this measure of magnetic ordering, the p-level anisotropy[3].

Rare earth metals are well known for exhibiting pronounced multiplet splittings of the $4 \mathrm{~d}$ levels[8-11], $4 \mathrm{f}$ levels $[10,12-15]$ and $4 \mathrm{~s}$ and $5 \mathrm{~s}$ levels $[10,16-17]$. Here we report, for the first time, the multiplet splittings of the Gd and $\mathrm{Tb} 5 \mathrm{p}$ levels and provide an explanation for their origin. As expected, the $5 p$ photoemission fine structure due to multiplet splittings is a result of the unfilled $4 \mathrm{f}$ and $5 \mathrm{~d}$ levels. We have undertaken our preliminary analysis by considering $4 f^{7}$ only for convenience. Contributions from the $5 \mathrm{~d}$ levels would not be expected to affect the number of multiplets, though this may seriously affect the multiplet oscillator strengths.

\section{EXPERIMENTAL}

The angle resolved photoemission experiments were carried out on a system described elsewhere $[2]$ on a $6 \mathrm{~m}$ toroidal grating monochrometer at the Synchrotron Radiation Center in Stoughton, Wisconsin. The photoelectrons were collected normal to the surface. The incident light of 37 or 70 degrees off normal was used to provide larger portion of light with its vector potential parallel to or perpendicular to the thin film (s- or p-polarization respectively). The combined energy resolution for photoemission was $0.25 \mathrm{eV}$.

Gd and $\mathrm{Tb}$ were deposited at room temperature onto $\mathrm{W}(110)$ and $\mathrm{Ni}(111)$ substrates respectively. The methods for preparing clean rare earth films have been described previously[1,2]. For $\mathrm{Gd} / \mathrm{W}(110)$, the base pressure was normally $7 \times 10^{-11}$ torr and the maximum during the deposition was $2 \times 10^{-10}$ torr, while for $\mathrm{Tb} / \mathrm{Ni}(111)$, the pressure was less than $5 \times 10^{-10}$ torr during the evaporation. The thickness was determined by a quartz crystal oscillator. The relative thickness has small error of $<2 \%$ while the absolute value can only be taken as nominal thickness. 1-3 monolayer Gd films on W(110) show sharp LEED pattern indicating the epitaxial growth with (0001) orientation of the hexagonal Gd overlayer. $\mathrm{Tb} / \mathrm{Ni}(111)$ films were not well-ordered. 


\section{RESULTS}

Photoemission spectra of the Gd $5 \mathrm{p}$ features were taken at $50 \mathrm{eV}$ and $60 \mathrm{eV}$ for $\mathrm{Gd}$ films on W(110) of different thicknesses. Fig. 1a is one of the typical spectra which include two main features with binding energies of about 21 and $28 \mathrm{eV}$ below the Fermi energy. For the films thicker than one monolayer, $21 \mathrm{eV}$ photoemission feature exhibits fine structure as shown in Fig. 1a. The envelope can be fitted well with four Gaussian peaks with binding energies of $20.3 \pm 0.1 \mathrm{eV}, 20.9 \pm 0.1 \mathrm{eV}, 22.0 \pm 0.2 \mathrm{eV}$, and $23.4 \pm 0.3 \mathrm{eV}$ (Fig.2a). $\mathrm{Gd}$ valence band spectra (not shown here and discussed in detail elsewhere[18]) shows 5d bands at $0.2 \pm 0.1 \mathrm{eV}$ and $1.3 \pm 0.1 \mathrm{eV}$. The $4 \mathrm{f}$ levels are observed at $8.6 \pm 0.1 \mathrm{eV}$. It should be noted that the FWHM of the $4 \mathrm{f}$ is $1 \mathrm{eV}$ and that of $5 \mathrm{~d}$ about $0.5 \mathrm{eV}$.

The $5 \mathrm{p}$ levels of $\mathrm{Tb} / \mathrm{Ni}(111)$ are shown in Fig. 1b. There are two main features with binding energies of 22 and $28 \mathrm{eV}$ respectively. As with Gd, the lower binding energy feature contains several peaks. The binding energies of these fine structures are $20.7 \mathrm{eV}$, $21.6 \mathrm{eV}, 22.7 \mathrm{eV}$, and $23.7 \mathrm{eV}$, as seen in Fig. $2 \mathrm{~b}$.

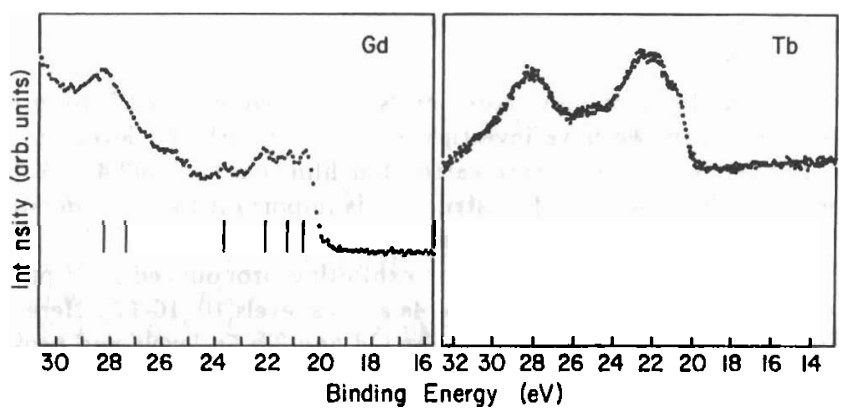

Fig. 1. (a) A typical Gd $5 p$ level photoemission spectrum with photon energy of $50 \mathrm{ev}$. (b) $\mathrm{Tb} 5 \mathrm{p}$ level photoemission spectrum with the photon energy of $60 \mathrm{ev}$.

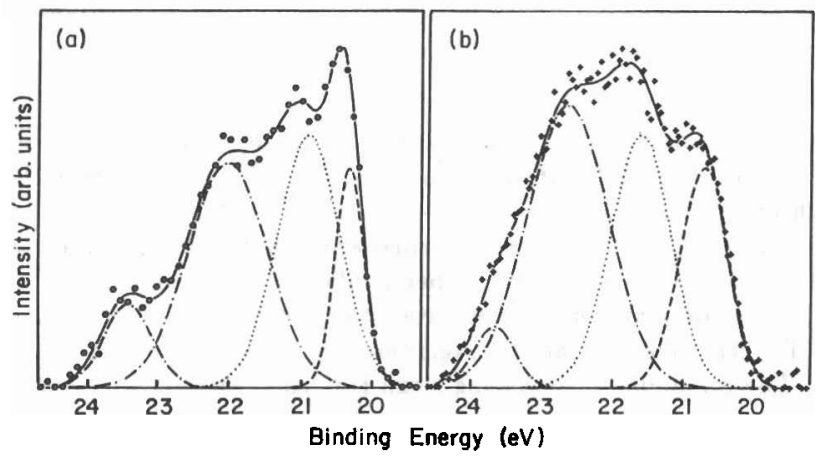

Fig. 2. The lower binding energy envelopes in Fig. 1. are fitted with four Gaussian functions after the linear background subtraction. The solid lines are the fitting result while the other lines showing the individual Gaussian function.

\section{DISCUSSION}

The large energy separation $(\sim 7 \mathrm{eV})$ of the two main features has been observed and is considered a result of spin-orbit coupling $[10,19]$. This value for the spin-orbit $\mathrm{Gd}$ 
$5 p$ coupling is larger than the calculated result of $4.0 \mathrm{eV}$ for atomic Gd[20]. Spin-orbit coupling only predicts two features, i.e. $5 p_{1 / 2}$ and $5 p_{3 / 2}$, without any further detailed structure.

Since rare earth atoms have unpaired $4 \mathrm{f}$ and $5 \mathrm{~d}$ electrons, multiplet structures, caused by the interaction between the core level photohole and those partly filled subshell, can have a strong influence on the core level spectra. Multiplet structures can be understood as a final state effect in photoemission. After one 5 p electron is excited, the hole left over will have an electrostatic interaction (direct, i.e. Coulumb interaction and indirect, i.e. exchange interaction) with the electrons in unfilled subshells to form different final states. This effect has been well studied on filled $s$ and $p$ core levels of some transition metals and rare earth $4 \mathrm{~s}, 5 \mathrm{~s}$, and $4 \mathrm{f}$ levels|15-17, 21-23|. Most of multiplet studies have only considered the exchange interaction between the unpaired spins which results in two main features characterized by the hole spin parallel or anti-parallel to that of the unpaired electrons. Configuration interactions were proven to be an important addition to this simple picture [23-24] and may cause further splitting in photoemission spectra. For s-levels, there is no spin-orbit interaction since they have no orbital momentum(l=0). For p-levels $(l=1)$, spin-orbit interaction can be ignored only when it is much weaker than the exchange interaction between the hole and other electrons, that is, in L-S coupling limit.

With L-S coupling scheme, we can work out the possible multiplets for Gd. The ground state configuration of $\mathrm{Gd}$ atom is $4 \mathrm{f}^{7} 5 \mathrm{~s}^{2} 5 \mathrm{p}^{6} 5 \mathrm{~d}^{1}$. Since $5 \mathrm{~d}^{1}$ is mainly polarized by $4 \mathrm{f}$ electrons, here we only write down the coupling of $5 p$ hole and $4 f\left({ }^{8} S_{7 / 2}\right)$ electrons for convenience. The existence of $5 \mathrm{~d}^{1}$ electrons should not affect the basic result of the following discussion as indicated later in this paper. Photoemission of one $5 p$ electron leaves an ion in $5 p^{5} 4 f^{7}$ configuration, which is complementary to $5 p^{1} 4 f^{7}$ (one $5 p$ hole and seven 4 electrons). Since $l=1, s=1 / 2$ for ${ }^{2} \mathrm{P}$ and $L^{\prime}=0, S^{\prime}=7 / 2$ for ${ }^{8} S$, L-S coupling of $5 p^{1}\left({ }^{2} P\right)$ and $4 f^{7}\left({ }^{8} S\right)$ results in ${ }^{7} P_{4,3,2}$ and ${ }^{9} P_{3,4,5}$ final states with $L=L^{\prime}+1, L '+1-1, \ldots$ , $\left|\mathrm{L}^{\prime}-1\right|, \mathrm{S}=\mathrm{S}^{\prime}+\mathrm{s}, \mathrm{S}^{\prime}+\mathrm{s}-1, \ldots,\left|\mathrm{S}^{\prime}-\mathrm{s}\right|$ and $\mathrm{J}=\mathrm{L}+\mathrm{S}, \mathrm{L}+\mathrm{S}-1, \ldots,|\mathrm{L}-\mathrm{S}|$. Without considering spin-orbit coupling, the two main features should be assigned as ${ }^{7} \mathrm{P}$ and ${ }^{9} \mathrm{P}$ with the later one at the lower binding energy. If weak spin-orbit coupling exists, the degeneracy of different $J$ values should be lifted and $21 \mathrm{eV}$ feature should split to three features of ${ }^{9} \mathrm{P}_{3}$, ${ }^{8} \mathrm{P}_{4},{ }^{9} \mathrm{P}_{5}$. Experimentally, we observe a four-fold splitting. Configuration interaction can cause further splitting of the photoemission features. Since ${ }^{9} \mathrm{P}$ term can only come from one configuration with all the spins of $5 \mathrm{p}$ hole and $4 \mathrm{f}$ electrons parallel, ${ }^{9} \mathrm{P}$ cannot further split and cannot exhibit fine structure beyond the three basic features. Since L-S theory predicts only three features and we observe four in our experiments(Fig.2), configuration interaction combined with L-S coupling scheme cannot be used to explain the observed data.

Alternatively, $\mathrm{j}-\mathrm{J}$ coupling can also result in multiplet splitting. Gupta and Sen[25] considered spin-orbit coupling in their multiplet calculations of $\mathrm{Mn}^{2+} 2 \mathrm{p}$ and $3 p$ levels and realized that spin-orbit coupling can be ignored for the $\mathrm{Mn} 3 p$ levels but it is the major factor in determining the photoemission structure of the $2 p$ levels. Their calculations are qualitatively confirmed by experiment|23]. In rare earth $5 p$ levels, spin-orbit coupling is strong and is larger for the heavier rare earths than the light rare earths. With strong spinorbit coupling, the two main features should be assigned as $5 p_{1 / 2}$ - and $5 p_{3 / 2}$-based levels with the latter one at lower binding energy. The relatively weaker exchange interaction of $5 p$ hole with $4 f$ and $5 d$ electrons will lift the J-degeneracy. The $j=3 / 2$ term couples with ${ }^{8} \mathrm{~S}_{7 / 2}$ to form four terms of $(3 / 2,7 / 2)_{5},(3 / 2,7 / 2)_{4},(3 / 2,7 / 2)_{3}$, and $(3 / 2,7 / 2)_{2}$ since $J=J^{\prime}+j, J^{\prime}+j-1, \ldots,\left|J^{\prime}-j\right|$. This is consistent with the four-fold splitting observed by 
experiment. Since L-S coupling and $\mathrm{j}-\mathrm{J}$ coupling should give out the same J-state energy order[26], we can assign the features with $\mathrm{J}$ quantum numbers as shown in Fig. 3. As Kowalczyk et al.[23] pointed out for Mn 2p levels, this splitting will result in an increase in the spin-orbit splitting, which could explain the increase of the splitting between the $5 \mathrm{p}_{1 / 2}$ and $5 \mathrm{p}_{3 / 2}$ levels $(7 \mathrm{ev})$ compared with theoretical spin-orbit splitting calculation $(4 \mathrm{ev})$.

Fig. 3. The order of different J-terms in L-S and $\mathrm{j}-\mathrm{J}$ coupling schemes

Whether L-S coupling or $\mathrm{j}-\mathrm{J}$ coupling occurs is totally dependent on the strength of different interactions. It is not surprising that most s-level multiplets fit L-S coupling multiplet theory very well. For p-levels $(\mathrm{l}=1)$, Mn $2 p$ levels are better explained by $\mathrm{j}-\mathrm{J}$ coupling while the $3 p$ levels, with weaker spin-orbit coupling, agrees with L-S coupling qualitatively $[23,25]$. Considering the magnititude of the two energy splittings caused by the two kinds of interaction (about $7 \mathrm{eV}$ and $1 \mathrm{eV}$ in our experiment), it is likely that a coupling intermediate between L-S and $\mathrm{j}-\mathrm{J}$ occurs in the case of Gd $5 \mathrm{p}$ multiplets.

Several other effects may also considered as the possible origin of the $5 p$ fine structure. Possible chemical shift, multiple valences, shake up or shake off effects can cause splittings in valence bands as well as in core levels. The necessary kinds of splittings are not observed, however, for the valence band[18] excluding these effects as the source of the shallow $5 \mathrm{p}$ core level fine structure in photoemission. Surface to bulk core level shifts have been observed in Gd $4 \mathrm{f}$ level of $0.5 \mathrm{eV}[12]$. We can expect a similar magnitude of shift for the $5 p$ levels. This effect cannot explain the energy separation of at least $0.7 \mathrm{eV}$ between $5 p$ fine structure features and the observation of four, instead of two features in the $5 \mathrm{p}_{3 / 2}$ photoemission envelope.

The existence of magnetic field can also cause the lifting of degeneracy in $5 \mathrm{p}_{3 / 2}$ and also forms four features with $\mathrm{m}_{j}=3 / 2,1 / 2,-1 / 2,-3 / 2$. This is, however, not likely to occur with a splitting as large as observed by experiment, nor can it explain the observed photoemission fine structure. This kind of magnetic field induced splitting should result in equal energy separations of $\Delta \mathrm{E}=\mathrm{g} \mu_{B} \mathrm{H}$. This is not observed in the experimental results. Estimating the magnetic field necessary to produce an energy splitting shown in the experiments $(\sim 1 \mathrm{eV})$ with $\Delta \mathrm{E} \sim \mu_{B} \mathrm{H}$, we find $\mathrm{H} \sim 10^{8}$ Gs. This is inconsistent with any reasonable external field produced by the rare earth film. If the field is produced by a magnetic dipole, the mean distance between the dipole and $5 p$ electron should be about $10^{-10} \mathrm{~cm}$. This is much smaller than the average distance between $4 \mathrm{f}$ and $5 \mathrm{p}$ or $5 \mathrm{~d}$ and $5 p$ electrons. Thus the local magnetic field produced by $4 f$ and $5 d$ magnetic moments cannot result in the observed photoemission fine structure of the rare earth $5 p$ levels.

Possible crystal field effects has also been considered. Since Gd films show hexagonal structure, we treat $5 p_{1 / 2}$ and $5 p_{3 / 2}$ levels with a crystal double group based on $D_{6}$ point group $\left(\mathrm{D}_{6}{ }^{\prime}\right)$ (Table I) similar to those derived by Tinkham[27] for cubic crystal. Comparing the character values of $\mathrm{p}_{1 / 2}$ and $\mathrm{p}_{3 / 2}$ levels with $\mathrm{D}_{6}{ }^{\prime}$ character table, we get $\mathrm{p}_{1 / 2} \longrightarrow \mathrm{E}_{3}$ 
$\mathrm{p}_{3 / 2} \longrightarrow \mathrm{E}_{3}+\mathrm{E}_{5}$

This means that $5 \mathrm{p}_{1 / 2}$ should not split while $5 \mathrm{p}_{3 / 2}$ should split to two features. This effect itself cannot explain the four-fold splitting. Furthermore, since crystal field effect is basically electrostatic interaction caused by a periodically distributed charge density, it should be stronger for the outer levels. However, Gd 5d feature has only FWHM of 0.5 $\mathrm{eV}$ at normal emission. We conclude that crystal field effects do not play an important rale in determining rare earth $5 p$ fine structure, although we cannot eliminate crystal field contribution completely.

Table I. The character table of the crystal double group $\mathrm{D}_{6}^{\prime}$ (the first 9 lines) and the corresponding characters of the rotation groups $\mathrm{D}_{\mathrm{J}}$ with $\mathrm{J}=1 / 2$ and $\mathrm{J}=3 / 2$ (the last 2 lines).

\begin{tabular}{|c|c|c|c|c|c|c|c|c|c|c|}
\hline & $D_{6}^{\prime}$ & E & $\mathbf{R}$ & $\mathrm{C}_{2}+\mathrm{RC}_{2}$ & $2 C_{3}$ & $2 \mathrm{RC}_{3}$ & $2 \mathrm{C}_{6}$ & $2 \mathrm{RC}_{6}$ & $3 \mathrm{C}_{2}^{\prime}+3 \mathrm{RC}_{2}^{\prime}$ & $3 \mathrm{C}_{2}^{\prime \prime}+3 \mathrm{RC}_{2}^{\prime \prime}$ \\
\hline \multirow{4}{*}{ 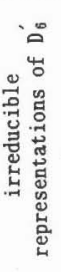 } & $\begin{array}{l}A_{1} \\
A_{2} \\
B_{1} \\
B_{2} \\
E_{1} \\
E_{2}\end{array}$ & $\begin{array}{l}1 \\
1 \\
1 \\
1 \\
2 \\
2\end{array}$ & $\begin{array}{l}1 \\
1 \\
1 \\
1 \\
2 \\
2\end{array}$ & $\begin{array}{r}1 \\
1 \\
-1 \\
-1 \\
-2 \\
2\end{array}$ & $\begin{array}{r}1 \\
1 \\
1 \\
1 \\
-1 \\
-1\end{array}$ & $\begin{array}{r}1 \\
1 \\
1 \\
1 \\
-1 \\
-1\end{array}$ & $\begin{array}{r}1 \\
1 \\
-1 \\
-1 \\
1 \\
-1\end{array}$ & $\begin{array}{r}1 \\
1 \\
-1 \\
-1 \\
1 \\
-1\end{array}$ & $\begin{array}{r}1 \\
-1 \\
1 \\
-1 \\
0 \\
0\end{array}$ & $\begin{array}{r}1 \\
-1 \\
1 \\
-1 \\
0 \\
0\end{array}$ \\
\hline & $\mathrm{E}_{3}$ & 2 & -2 & 0 & 1 & -1 & $\sqrt{3}$ & $-\sqrt{3}$ & 0 & 0 \\
\hline & $E_{4}$ & 2 & -2 & 0 & 1 & -1 & $-\sqrt{3}$ & $\sqrt{3}$ & 0 & 0 \\
\hline & $\mathrm{E}_{5}$ & 2 & -2 & 0 & -2 & 2 & 0 & 0 & 0 & 0 \\
\hline \multicolumn{2}{|c|}{$j=1 / 2$} & 2 & -2 & 0 & 1 & -1 & $\sqrt{3}$ & $-\sqrt{3}$ & 0 & 0 \\
\hline \multicolumn{2}{|c|}{$j=3 / 2$} & 4 & -4 & 0 & -1 & 1 & $\sqrt{3}$ & $-\sqrt{3}$ & 0 & 0 \\
\hline
\end{tabular}

It should be noted that although we did not consider $5 \mathrm{~d}^{1}$ electron in our multiplet analysis while writing out the final state terms, it is likely that $5 \mathrm{~d}$ electrons have large contribution to $5 \mathrm{p}$ multiplets since they have the same principle quantum number. The $\mathrm{j}-\mathrm{J}$ coupling of $5 \mathrm{~d}^{1}\left({ }^{2} \mathrm{D}_{5 / 2}\right)$ and $5 \mathrm{p}^{1}\left({ }^{2} \mathrm{P}_{3 / 2}\right)$ results in four terms of $\mathrm{J}=4,3,2$, and 1 , which is also correspond to a four-fold splitting as we consider the coupling with $4 \mathrm{f}$ only. Further experiments and detailed calculations are needed to determine the portion of $5 \mathrm{~d}$ contribution.

$\mathrm{Tb}$ has ground state configuration of $4 \mathrm{f}^{8} 5 \mathrm{~s}^{2} 5 \mathrm{p}^{6} 5 \mathrm{~d}^{1}$. This is similar to that of Gd though $\mathrm{Tb}$ has only six unpaired $4 \mathrm{f}$ electrons instead of seven in Gd. Most of the above discussion is valid for $\mathrm{Tb}$. The fine structure can again be explained as multiplets under $\mathrm{j}-\mathrm{J}$ coupling or intermediate coupling scheme.

\section{CONCLUSION}

We have observed the photoemission fine structure splitting the $5 p$ levels for $\mathrm{Gd}$ and $\mathrm{Tb}$. Our analysis suggests that multiplet structures arise as result of $\mathrm{j}$-J coupling or intermediate coupling scheme. Since $j-J$ coupling is more complicated than L-S coupling, more theoretical calculations considering spin-orbit coupling, exchange interaction, configuration interaction, and crystal field effects are necessary to fully understand this effect. Since the fine structure is caused by exchange interaction of the unpaired electrons, it has the potential to be used to study rare earth $4 \mathrm{f}$ and $5 \mathrm{~d}$ unpaired spins and may give us some insights to many interesting problems such as local spin and mixed valence. 


\section{ACKNOWLEDGEMENTS}

The authors would like to thank Rong-Tzong Wu, D.LaCraffe, and C.Hwang for their technical assistance. This work was funded by the U.S.DOE through grant no. DE-FG02-90ER45319. The experiments were conducted at the Synchrotron Radiation Center in Stoughton, Wisconsin, which is funded by the NSF.

\section{REFERENCES}

1. D.LaGraffe, P.A.Dowben, and M.Onellion, Phys. Rev. B40, 970 (1989).

2. P.A.Dowben, D.LaGraffe, and M.Onellion, J. Phys. Cond. Matt., 1, 6751 (1989).

3. D.LaGraffe, P.A.Dowben, and M.Onellion, Phys. Lett. A147, 240 (1990).

4. P.A.Dowben, D.LaGraffe, Dongqi Li, A.Miller, Ling Zhang, L.Dottl, and M.Onellion, Phys. Rev. B43, 3171 (1991).

5. C.S.Fadley, in Magnetic Properties of Low Dimensional Systems II, Springer Proceeding in Physics, vol.50, Edited by L.M.Falicov, $\overline{\mathrm{F}} . \bar{M}$.jia-Lira, and J.L.Moranlopez, (Springer-Verlag, Berlin, 1990), p.36; Physica Scripta T17, 39 (1987).

6. B.Sinkovic and C.S.Fadley, Phys. Rev. B31, 4665 (1985).

7. B.Sinkovic, B.Hermsmeier and C.S.Fadley, Phys. Rev. Lett., 55, 1227 (1985).

8. C.S.Fadley and D.A.Shirley, Phys. Rev. A2, 1109 (1970).

9. S.P.Kowalczyk, N.Edelstein, F.R.McFeely, L.Ley and D.A.Shirley, Chem. Phys. Lett., 29, 491 (1974).

10. B.D.Padalia, W.C.Lang, P.R.Norris, L.M.Watson, and D.J.Fabian, Proc. R. Soc. Lond., 354, 269 (1977).

11. J.L.Dehmer, A.F.Starace, U.Fano, J.Sugar, and J.W.Cooper, Phys. Rev, Lett., 26, 1521 (1971).

12. R.Kammerer, J.Barth, F.Gerken, A.Flodström and L.I.Johansson, Solid State Commun., 41, 435 (1982).

13. Y.Baer and G.Busch, J. Electron. Spectrosc. Rel. Phenom., 5, 611 (1974).

14. J.K.Lang, Y.Baer and P.A.Cox, J. Phys. F11, 121 (1981).

15. P.A.Cox, J.K.Lang and Y.Baer, J, Phys. F11, 113 (1981).

16. R.L.Cohen, G.K.Wertheim, A.Rosenewaig and H.J.Guggenheim, Phys. Rev. B5, 1037 (1972).

17. F.R.McFeely, S.P.Kowalczyk, L.Ley and D.A.Shirley, Phys. Lett. 49A, 301 (1974).

18. Dongqi Li, C.Hutchings, P.A.Dowben, Rong-Tzong Wu, C.Hwang, M.Onellion, B.Andrews and J.L.Erskine, in preparation.

19. G.K.Wertheim, R.L.Cohen, A.Rosenewaig, H.J.Guggenheim, in Electron Spectroscopy, Edited by D.A.Shirley (Amsterdan: North Holland, 1972), p.813.

20. F.Herman and S.Skillman, Atomic Calculations, (Prentice-Hall, Englewood Cliff, N.J., 1963).

21. C.S.Fadley, D.A.Shirley, A.J.Freeman, P.S.Bagus, J.V.Mallow, Phys. Rev. Lett., 23, 1397 (1969).

22. B.Hermsmeier, C.S.Fadley, M.O.Krause, J.Jimenez-Mier, P.Gerard, S.T.Manson, Phys. Rev. Lett., 61, 2592 (1988).

23. S.P.Kowalczyk, L.Ley, F.R.McFeely and D.A.Shirley, Phys. Rev. B11, 1721 (1975).

24. P.S.Bagus, A.J.Freeman and F.Sasaki, Phys. Rev. Lett., 30, 850 (1973).

25. R.P.Gupta and S.K.Sen, Phys. Rev. B10, 71 (1974); Phys. Rev. B12, 15 (1975).

26. H.E. White, Introduction to Atomic Spectra, (McGraw-Hill Book Company, 1934).

27. M.Tinkham, Group Theory and Quantum Mechanics, (McGraw-Hill Publishing Company, 1964), p.77. 